Marquette University

e-Publications@Marquette

$5-2019$

\title{
Establishment and Replication of Emotion Regulation Profiles: Implications for Psychological Health
}

Samantha Chesney

Marquette University

Sydney Clare Timmer-Murillo

Marquette University

Nakia Gordon

Marquette University, nakia.gordon@marquette.edu

Follow this and additional works at: https://epublications.marquette.edu/psych_fac

Part of the Psychology Commons

\section{Recommended Citation}

Chesney, Samantha; Timmer-Murillo, Sydney Clare; and Gordon, Nakia, "Establishment and Replication of Emotion Regulation Profiles: Implications for Psychological Health" (2019). Psychology Faculty Research and Publications. 474.

https://epublications.marquette.edu/psych_fac/474 
Marquette University

e-Publications@Marquette

\section{Psychology Faculty Research and Publications/College of Arts and Sciences}

This paper is NOT THE PUBLISHED VERSION; but the author's final, peer-reviewed manuscript. The published version may be accessed by following the link in the citation below.

Anxiety, Stress \& Coping, Vol. 32, No. 3 (May 2019): 329-345. DOI. This article is (C) Routledge Taylor \& Francis Group and permission has been granted for this version to appear in e-

Publications@Marquette. Routledge Taylor \& Francis Group does not grant permission for this article to be further copied/distributed or hosted elsewhere without the express permission from Routledge Taylor \& Francis Group.

\section{Establishment and Replication of Emotion Regulation Profiles: Implications for Psychological Health}

Samantha A. Chesney

Department of Psychology, Marquette University, Milwaukee, WI

Sydney C. Timmer-Murillo

Department of Psychology, Marquette University, Milwaukee, WI

Nakia S. Gordon

Department of Psychology, Marquette University, Milwaukee, WI

\section{Abstract}

Background and Objectives: A promising method of capturing the complex nature of emotion regulation is to assess composite profiles of regulation (i.e., default pattern of regulation across

multiple strategies). However, it remains unclear whether regulatory profiles demonstrate consistency across samples and in relation to mental health. Design: Two studies are presented here. Both utilized 
a cross-sectional design, and the second study presents a replication of the first. Method: Both studies utilized self-report data from independent undergraduate samples to perform latent profile analyses of emotion regulation use. Results: Studies 1 and 2 demonstrated evidence for four replicable regulatory profiles: Adaptive, Accepting (with or without Suppression), Non-accepting, and Maladaptive. Profiles were also related to symptoms of depression and anxiety, such that those consistently relying on adaptive strategies reported lower symptoms than those relying more heavily on maladaptive strategies. Conclusions: These findings clarify previous work which tied regulatory profiles to psychological health by extending a person-centered approach to understanding the ways in which individuals regulate their emotions.

\section{Keywords}

Emotion regulation, profiles, repertoire, psychopathology, latent profile analysis, person-centered

It is long understood that the myriad ways individuals regulate their emotional experiences are related to varying degrees of psychological wellness (Gross, 2002; Lazarus, 1991). Accordingly, researchers in affective science have set out to empirically identify which regulatory strategies lead to desired psychological outcomes and which typically have undesired results. For example, meta-analytic results indicate that greater engagement in problem solving and cognitive reappraisal to regulate emotion is associated with less psychological distress (i.e., fewer anxiety and depressive symptoms), whereas greater reliance on avoidance, expressive suppression, and rumination are related to increased symptom levels (Aldao, Nolen-Hoeksema, \& Schweizer, 2010). These one-to-one relationships connecting a single emotion regulation strategy to a set of psychological symptoms established an empirical foundation and, for a while, appeared to represent the likely outcomes of regulating in a specific way. However, continued fervor gave rise to questioning whether a single strategy is actually "adaptive" or "maladaptive" across all contexts. For example, studies of expressive suppression (Le \& Impett, 2013) and rumination (Kashdan, Young, \& McKnight, 2012) suggest that these strategies can actually be beneficial to well-being in certain contexts, despite their reputations as "maladaptive". Additionally, the putatively "adaptive" strategy of cognitive reappraisal can actually lead to greater cognitive depletion in certain contexts (Keng, Robins, Smoski, Dagenbach, \& Leary, 2013).

Findings that evidence such notable contradictions suggest that the way in which an individual's psychological state is related to their emotion regulation habits is not effectively captured by assessing the use of a single strategy in isolation. Indeed, experience sampling studies show that people rely on diverse regulatory strategies in day-to-day life. For instance, Brans, Koval, Verduyn, Lim, and Kuppens (2013) asked participants to report on their naturalistic regulation of emotion with six emotion regulation strategies at 10 timepoints over a period of seven days. Results from initial and independent replication samples indicated strong positive, within-person correlations between all of the strategies, thus providing evidence that individuals rely on several strategies, rather than only one or two, to regulate throughout the day. Further support was found by Heiy and Cheavens (2014), who assessed participants naturalistic regulation three times per day for 10 days. Regulation of positive and negative emotion were assessed independently of each other, and the use of 20 strategies were evaluated in each; thus, a total of 40 strategies were assessed at each timepoint. Their results indicated that 
individuals use an average of 7.2 strategies to regulate a single negative experience and an average of 7.6 strategies to regulate a single positive experience.

Beyond determining the number of regulatory strategies a person uses throughout the day, or for a single event, studies also demonstrate that the interplay between strategies is meaningful for psychosocial outcomes. Aldao and Nolen-Hoeksema (2012) conducted a cross-sectional study of community adults that showed engagement in "adaptive" strategies was only associated with fewer symptoms of depression, anxiety, and substance abuse when maladaptive regulation was also high. In a later study investigating social anxiety, those researchers suggest that the benefits of adaptive strategies were often more effective at reducing symptoms when participants were also using high levels of maladaptive strategies, indicating that the benefits of adaptive strategies may be less potent when use of maladaptive strategies is low (Aldao, Jazaieri, Goldin, \& Gross, 2014). While it should be noted that the interaction of strategies is not universally consistent [e.g., highly symptomatic individuals do not necessarily benefit from the interplay; see Aldao et al. (2014)], this does not detract from the conclusion that regulatory strategies influence each other in consequential ways.

Thus, a comprehensive understanding of psychosocial outcomes from emotion regulation cannot be distilled down to a regression that accounts for only one strategy, nor can it result from investigation methods that evaluate multiple strategies as isolated, independent processes. Instead, researchers acknowledge the benefits of employing person-centered approaches (Dixon-Gordon, Aldao, \& De Los Reyes, 2015; Doré, Silvers, \& Ochsner, 2016; Gabriel, Daniels, Diefendorff, \& Greguras, 2015). These methods attend to the individual as the unit of study and create "personalized" models of emotion regulation (Aldao, 2013; Aldao \& Tull, 2015; Doré et al., 2016), which identify the outcomes of regulation by taking into account the dynamic interactions between the person and the wide array of regulatory strategies available to them. In contrast to a variable-centered approach that identifies relationships between predictors and outcomes separately across people, person-centered approaches utilize constellations of variables, working as a system within people, to assess multifactorial relationships. Thus, a person-centered approach has the power to characterize the use of many emotion regulation strategies - capturing not only how strategies are used, but how they are used in the context of other strategies - and more appropriately represent the interplay of a person's regulation processes.

Person-centered approaches have been used to identify distinct groups of adults who can be differentiated by how frequently they employ a variety of regulatory strategies to manage their emotions. These groups are said to have particular profiles (alternatively termed "patterns" or "repertoires") of emotion regulation (Chesney \& Gordon, 2017; De France \& Hollenstein, 2017; DixonGordon et al., 2015; Eftekhari, Zoellner, \& Vigil, 2009; Van Eck, Warren, \& Flory, 2017). Thus far, the literature consistently demonstrates the presence of three, replicable profiles: ( 1 ) a profile that tends towards putatively adaptive strategies and higher psychological wellness; ( 2 ) a profile that tends towards putatively maladaptive strategies and lower psychological wellness; and ( 3 ) a profile that is more balanced in its proportion of adaptive to maladaptive strategy use (for example, high or low use across all strategies) with varying levels of psychological wellness.

The earliest emotion regulation profile study (Eftekhari et al., 2009) assessed two strategies, cognitive reappraisal and expressive suppression, to develop profiles predicting psychopathology in 
undergraduate students. Using cluster analyses, the authors found four emotion regulation profiles: (1) Low Regulators (i.e., individuals who report low reappraisal and moderate suppression); ( 2) High Regulators (i.e., individuals who often use both reappraisal and suppression); ( 3) High Reappraisers/Low Suppressors; and (4) Moderate Reappraisers/Low Suppressors. Expanding the research, Dixon-Gordon et al. (2015) used a latent class analysis (LCA) of seven emotion regulation strategies to identify five profiles in undergraduates, and Chesney and Gordon (2017) conducted a cluster analysis of six emotion regulation strategies to establish four profiles in a community sample. In each of these three studies, a profile was found that was characterized by participants' disproportionately high use of what are broadly considered adaptive strategies, including the "Adaptive" regulators (i.e., high use of acceptance, reappraisal, and problem solving; Chesney \& Gordon, 2017; Dixon-Gordon et al., 2015) and the "High Reappraisers/Low Suppressors" (Eftekhari et al., 2009). Notably, participants with each of these profiles demonstrated minimal psychological distress. The presence of high-symptom, maladaptive profile patterns was also corroborated by these studies, including the Maladaptive Regulators profile (i.e., high use of avoidance, suppression, and rumination; Chesney \& Gordon, 2017) and the Worriers/Ruminators profile (i.e., high use of worry and rumination; Dixon-Gordon et al., 2015).

Dixon-Gordon et al. (2015) also found evidence of Low Regulators (i.e., below-average use of all strategies) and High Regulators (i.e., above-average use of all strategies). However, whereas Eftekhari et al. (2009) found that their Low Regulators reported greater psychopathology, Dixon-Gordon et al. (2015) showed the opposite finding: High Regulators had higher levels of psychological distress. This inconsistency may be explained by differences in study design. In the Eftekhari et al. (2009) study, "low regulator" meant low engagement in cognitive reappraisal and expressive suppression - it cannot necessarily be generalized to low engagement in all strategies. Indeed, studies including greater numbers of strategies do not find a low regulator group (Chesney \& Gordon, 2017; De France \& Hollenstein, 2017). Further, contrary to the name, Eftekhari et al. (2009) Low Regulators profile averaged significantly higher frequency of expressive suppression than two of the other profiles, putting it second only to the High Regulators group for use of this strategy. This moderately high level of suppression coupled with a low level of reappraisal is akin to a "maladaptive" profile pattern, which is consistent with this profile evidencing the highest symptom severity averages in this study.

The studies summarized here indicate that several profile types are expected - with some certainty - to be present in any random sample, including at least one profile that is dominated by strategies typically associated with desirable psychosocial outcomes (i.e., "adaptive") and at least one profile that is chiefly comprised of strategies typically associated with undesirable psychosocial outcomes (i.e., "maladaptive"). Other types, such as the Low and High Regulators profiles, have some inconsistencies that require investigators to better account for variability due to emotion regulation measurement (i.e., number and type of strategy), sample characteristics (e.g., age, level of psychological distress), and statistical analysis (i.e., cluster analysis versus latent modeling [see Statistical Analyses section]).

Recently, De France and Hollenstein (2017) addressed this concern with a two studies of emotion regulation profiles identified by latent profile analyses (LPA) of their recently developed measure, the Regulation of Emotion Systems Survey (RESS). The RESS assesses the use of six regulatory strategies, including distraction, rumination, cognitive reappraisal, expressive suppression, expressive 
engagement, and arousal control. The first study defined three regulatory profiles: Expressive Engagement Propensity (above-average use of emotional expression), Expressive Suppression Propensity (above-average use of expressive suppression), and Average Regulation (approximately average-level use across all strategies; De France \& Hollenstein, 2017). Analyses indicated that individuals with the Expressive Suppression profile endorsed more severe anxiety, depression, and difficulties with family/peer relationships than individuals who used all strategies at levels close to the sample mean (i.e., the Average Regulation group). The results of their second study replicated the presence of these three profiles and identified an additional Multi-strategy profile characterized by above-average use of all strategies. In the presence of this profile, symptom differences between emotion regulation profiles were generally non-significant; only the Average Regulation profile evidenced less family relationship difficulties than the Expressive Engagement profile. As the studies had significant procedural overlap, authors could rule out discrepancies in psychosocial outcomes due to factors such as number or type of strategies. This allowed the authors to narrow their proposals for differences to either a younger sample in the first study (i.e., late teens versus early 20's) and/or differences in survey completion methods (i.e., lab computer versus online at home; De France \& Hollenstein, 2017). Therefore, it is not yet known what outcomes would result from holding all critical methodological variables constant.

While promising, the existing literature is not sufficient to conclude whether a there are reliable relationships between regulatory strategies that result in a set of defined profiles, nor whether a particular profile is reliably related to particular outcomes, even without variability in methods and sample characteristics. To our knowledge, only one partial-replication study has yet been published and important profile replicability questions still demand attention. As such, the current studies fill a gap in the foundation for evidence of replicable profiles with a unique investigation that includes six, frequently studied emotion regulation strategies (acceptance, cognitive reappraisal, problem solving, avoidance, expressive suppression, and rumination) and uses the measurement instruments that are most commonly used to assess them. To identify these strategies and measures, we relied on a metaanalysis (Aldao et al., 2010) that reported these as being among the most well-defined regulatory strategies. Thus, they provide an apt target for research in this area. Results from two studies are presented. In the first study, an LPA was conducted to identify profiles of emotion regulation, and their corresponding levels of depression and generalized anxiety, in a typically healthy, undergraduate sample. The second study was conducted to determine whether the profiles and relationships to psychological outcomes from Study 1 could be replicated utilizing a similar sample and identical method. We predicted that the current samples will evidence a putatively adaptive regulation pattern (i.e., relatively frequent use of acceptance, cognitive reappraisal, and problem solving) that would be associated with low levels of psychological distress, as well as a putatively maladaptive regulation pattern (i.e., relatively frequent use of avoidance, expressive suppression, and rumination) that would be associated with heightened psychological distress. Additionally, we expected there to be a group of individuals whose regulatory profile was not predominantly adaptive or maladaptive, such as a high regulators group that often use many (or all) of the assessed strategies. Recent research indicates that individuals with this regulation style would have moderate symptom levels (Dixon-Gordon et al., 2015), though debate surrounding this hypothesis still exists. 


\section{Study 1}

Stemming from previous literature on profiles of emotion regulation, Study 1 was designed to establish regulatory profiles in a young adult sample. The aim was to investigate the person-centered differences in individuals who rely on specific strategies in concert with multiple other strategies. This will allow for a better understanding of how regulation choices correlate with anxiety and depression symptom severity.

\section{Method}

\section{Participants}

Undergraduate participants $(N=176)$ from an urban, Midwestern university were recruited via advertisements to participate in research for partial course credit. Participants currently taking psychotropic medications were informed that they must have achieved medication stabilization (i.e., compliance with a stable dose for at least six weeks). All participants underwent informed consent, and all procedures were approved by the Institutional Review Board of Marquette University, where procedures were completed.

The mean age for participants in this sample was 18.84 years $(S D=1.00)$, and $83 \%$ were female. The majority of participants identified as Caucasian ( $n=141 ; 80.11 \%)$, and the remainder of the sample identified as Hispanic/Latino ( $n=9 ; 5.11 \%)$, African American $(n=6 ; 3.41 \%)$, Asian $(n=5 ; 2.84 \%)$, or other (including multiracial individuals; $n=15,8.52 \%$ ).

\section{Materials}

All measures were administered in full; however, only the subscales specified below were used for analyses. A meta-analysis of emotion regulation (Aldao et al., 2010) was used to assist in the identification of a valid and reliable self-report measure for each strategy.

\section{Emotion Regulation Questionnaires.}

The Difficulties in Emotion Regulation Scale (DERS; Gratz \& Roemer, 2004) is a 36-item measure designed to assess several factors of emotion regulation. Responses to the Nonacceptance of Emotional Responses subscale ( 6 items) served as the measure of Acceptance use. Items of this scale measured the degree to which an individual accepts their own emotional experiences, such as "When I'm upset, I become angry with myself for feeling that way" and "When I'm upset, I feel guilty for feeling that way". To ease understanding of the results, this subscale was reverse scored (i.e., a higher score indicating more acceptance) to be consistent with means, standard deviations, and standardized scores of the profiles. Both subscales of the Emotion Regulation Questionnaire (ERQ; Gross \& John, 2003) were used. One subscale assessed typical use of Cognitive Reappraisal (6-item subscale), referring to changing one's thought processes when wanting to feel more or less positive or negative emotion (e.g., "I control my emotions by changing the way I think about the situation I'm in"). The Expressive Suppression (4-item subscale) assessed one's inhibition of outward emotional reactions and their efforts to not express emotion (e.g., "When I am feeling negative emotions, I make sure not to express them" and "I keep my emotions to myself"). The Coping Responses Inventory (CRI; Moos, 1993) is a 48item measure which contains multiple subscales related to managing distress via avoidance. The current study followed previous assessments (Chesney \& Gordon, 2017; Holahan, Moos, Holahan, Brennan, \& Schutte, 2005) of Avoidance by summing the Cognitive Avoidance (6 items; 
Cronbach's $\alpha=.72$ ) and Emotional Discharge ( 6 items; Cronbach's $\alpha=.51$ ) subscales of this measure to provide a more comprehensive assessment of varying avoidance techniques. These subscales included items such as, "Try to forget the whole thing", "Keep away from people in general", and "Take it out on other people when you feel angry or depressed". Thus, overall Avoidance indicated how often a respondent made both cognitive and behavioral attempts to avoid a stressor. The Problem

Solving subscale of the CRI (6 items) was used to assess the tendency to make a plan and, if necessary, make multiple attempts to follow through (e.g., "Try to learn to do more things on your own" and "Know what has to be done and try hard to make things work"). The Cognitive Emotion Regulation Questionnaire (CERQ; Garnefski \& Kraaij, 2006a) evaluated Rumination in the current sample (2 item subscale), assessing how often a respondent was thinking about, being preoccupied with, and wanting to understand how he/she feels about his/her experiences (e.g., "I am preoccupied with what I think and feel about what I have experienced.")

\section{Symptoms of Psychological Distress.}

The Beck Depression Inventory-II (BDI-II; Beck, Steer, \& Brown, 1996) is a validated 21-item self-report inventory commonly used to assess intensity of current depressive symptoms in undergraduate samples. A total depression score was obtained for each participant by summing the responses for all items. The Beck Anxiety Inventory (BAl; Beck, Epstein, Brown, \& Steer, 1988) is a 21-item self-report inventory designed to assess intensity of current anxiety symptoms. A total anxiety score was obtained by summing the responses for all items.

Procedure

Participants presented to a university laboratory, where they were tested independently. They first read and signed informed consent forms and had any questions answered by the research assistant. They then completed the study measures in one, approximately 60-minute session using computerized versions of the surveys. Measures were presented in the following order: demographics form, CERQ, BDI-II, DERS, BAI, ERQ, and CRI.

Statistical analyses

Latent profile analysis (LPA) provides an advanced person-centered method that is preferable to traditional cluster and regression analyses (Pastor, Barron, Miller, \& Davis, 2007). Specifically, this method was used in the current study because LPA is a model-based approach that provides fit statistics to guide investigators' decisions on the most appropriate profile solution, including number and structure of profiles present in the data. Additionally, this analysis provides the distinct probabilities of each participant being a member of each identified profile, therefore allowing investigators to confidently classify individuals. The current study statistically grouped participants into profiles of emotion regulation according to how frequently they used six emotion regulation strategies. [See Berlin, Williams, and Parra (2014) for an extended introduction to latent modeling.] Each regulatory profile identified by the LPA represents a group of individuals whose pattern of scores on observed indicator variables is relatively homogenous. The observed indicator variables in the current study included the six discrete emotion regulation subscales (acceptance, cognitive reappraisal, problem solving, avoidance, expressive suppression, and rumination).

Using an inductive approach to model generation, the first analysis specified a two-profile model. Then, iterative models were generated, adding one profile each time, until the increase in model fit no 
longer merited adding additional latent profiles (Nylund, Asparouhov, \& Muthén, 2007). To evaluate model fit, six statistics were examined: Akaike information criterion (AIC; Akaike, 1973), Bayesian information criterion (BIC; Schwarz, 1978), sample-size-adjusted BIC (SSA-BIC; Sclove, 1987), entropy, Vuong-Lo-Mendell-Rubin (VLMR) likelihood ratio test, and adjusted Lo-Mendell-Rubin likelihood ratio test (adjusted LMR; Lo, Mendell, \& Rubin, 2001). Fit for each model was evaluated in relation to the other models, and the profile solution with the best resulting combination of statistics was chosen. Lower numbers for the AIC, BIC, and SSA-BIC indicate a better fitting model (Nylund et al., 2007). Thus, increasing the number of profiles was considered if the values for these statistics were lower than the values for models with fewer profiles. Higher entropy values (i.e., values approaching 1) indicate better model classification of individuals into groups (Celeux \& Soromenho, 1996). Finally, the VLMR and adjusted LMR present likelihood ratio tests whereby significant $p$ values $(p<0.05)$ indicate that the tested model is a better fit than a model with one fewer group (Nylund et al., 2007). Given that this analysis provides the estimated probabilities for an individual belonging to each of the profiles, participants were coded as having the profile that had the highest probability of fitting with their individual regulation pattern. LPA analyses were run in Mplus ${ }^{\odot}$, Version 7.4 (Muthén \& Muthén, 19982017). All other analyses were conducted with SPSS Version 24.0. An alpha level of.05 was used to evaluate statistical probabilities.

\section{Results}

Variable screening did not reveal any outliers, non-normal distributions, or idiosyncratic response patterns; therefore, all participants were included in analyses. Missing data were mean imputed for symptom report measures (BDI, BAI) with an item response rate greater than or equal to $85 \%$; no symptom measures had an item response rate of less than $85 \%$. A maximum of one missing item was allowed for each emotion regulation subscale, for which the mean was then imputed. An exception was made for the Rumination subscale of the CERQ, for which all items were required due to scale brevity.

Descriptives and zero-order analyses

All demographics were assessed for an association with scores on emotion regulation subscales and the established regulatory profiles (see Profile Solution for descriptions). Analyses established that age, gender, and race were not significant covariates of emotion regulation outcomes in the current sample. Descriptive statistics of each subscale are presented in Table 1.

Table 1. Descriptive statistics on emotion regulation subscales and symptom measures.

\begin{tabular}{|l|l|l|l|l|l|}
\hline & $\mathrm{M}$ & SD & Range & Number of items & $\alpha$ \\
\hline Emotion regulation & & & & & \\
\hline Acceptance (DERS) & 23.84 & 5.15 & $6-30$ & 6 & .88 \\
\hline & 23.69 & 5.47 & & & .90 \\
\hline Cognitive reappraisal (ERQ) & 29.02 & 6.93 & $6-42$ & 6 & .88 \\
\hline & 30.03 & 6.49 & & & .88 \\
\hline Problem solving (CRI) & 10.67 & 3.45 & $0-18$ & 6 & .73 \\
\hline & 10.45 & 3.96 & & & .80 \\
\hline Avoidance (CRI) & 12.91 & 5.63 & $0-36$ & 12 & .73 \\
\hline
\end{tabular}




\begin{tabular}{|l|l|l|l|l|l|}
\hline & 13.71 & 5.50 & & & .72 \\
\hline Expressive suppression (ERQ) & 14.43 & 4.96 & $4-28$ & 4 & .74 \\
\hline & 14.87 & 5.19 & & & .79 \\
\hline Rumination (CERQ) & 5.44 & 2.04 & $2-10$ & 2 & .72 \\
\hline & 5.27 & 2.04 & & & .64 \\
\hline Psychopathology & & & & & \\
\hline Depression (BDI-II) & 11.88 & 9.41 & $0-63$ & 21 & .91 \\
\hline & 10.42 & 9.21 & & & .93 \\
\hline Generalized anxiety (BAI) & 11.93 & 11.68 & $0-63$ & 21 & .95 \\
\hline & 10.47 & 10.37 & & & .94 \\
\hline
\end{tabular}

Note: Study 1 sample descriptives are in boldface type; Study 2 sample descriptives are in regular type. DERS-Nonacceptance subscale was reverse coded such that higher numbers indicate more acceptance. The reported range depicts the total possible range of each scale.

Profile solution

Table 2 provides the fit statistics for all tested models. After evaluation of all model fit statistics, the four-profile model was determined to provide the best fit to the data. Specifically, this number of groups exhibited the lowest BIC value and has an entropy value (entropy $=0.79$ ) very near the cutoff of 0.80 for a high level of entropy (Clark \& Muthén, 2009). Thus, the four-profile solution classified $79.1 \%$ of individuals to the correct latent profile. Although the three-profile solution evidenced a higher entropy value, the VLMR and adjusted LMR indicated that the four-profile solution was a significantly better fit. While the five- and six-profile models exhibited lower values on the AIC and adjusted BIC, these small reductions are not supported by any other fit statistics and do not warrant increasing the number of latent profiles (Kass \& Raftery, 1995). Therefore, the four-profile structure was retained because it provided the best resolution of fit statistics and theoretical support.

Table 2. Fit statistics for LPA model structures.

\begin{tabular}{|l|l|l|l|l|l|l|l|}
\hline & $\begin{array}{l}\text { Number of } \\
\text { profiles }\end{array}$ & AIC & BIC & $\begin{array}{l}\text { Sample-size } \\
\text { adjusted BIC }\end{array}$ & Entropy & $\begin{array}{l}\text { VLMR } \\
\text { ( } p \text { value) }\end{array}$ & $\begin{array}{l}\text { Adjusted } \\
\text { LMR ( } p \text { value) }\end{array}$ \\
\hline Study 1 & & & & & & & \\
\hline $\mathrm{N}=176$ & 2 & 6062.495 & 6122.734 & 6062.566 & 0.889 & 0.0364 & 0.0394 \\
\hline & 3 & 6044.098 & 6126.531 & 6044.195 & 0.900 & 0.3096 & 0.3175 \\
\hline & 4 & 6013.103 & 6117.729 & 6013.226 & 0.791 & 0.0184 & 0.0206 \\
\hline & 5 & 6003.434 & 6130.254 & 6003.584 & 0.785 & 0.8158 & 0.8208 \\
\hline & 6 & 5997.732 & 6146.745 & 5997.907 & 0.766 & 0.3586 & 0.3620 \\
\hline Study 2 & & & & & & & \\
\hline $\mathrm{N}=147$ & 2 & 5106.581 & 5163.399 & 5103.273 & 0.784 & 0.0187 & 0.0208 \\
\hline & 3 & 5085.979 & 5163.731 & 5081.453 & 0.731 & 0.5198 & 0.5281 \\
\hline & 4 & 5071.145 & 5169.830 & 5065.400 & 0.711 & 0.6799 & 0.6829 \\
\hline & 5 & 5065.513 & 5185.130 & 5058.549 & 0.729 & 0.3075 & 0.3098 \\
\hline & 6 & 5059.850 & 5200.400 & 5051.667 & 0.798 & 0.0000 & 0.0000 \\
\hline & 7 & 5045.499 & 5206.982 & 5036.100 & 0.824 & 0.1867 & 0.1953 \\
\hline
\end{tabular}


Note: Bold indicates the model determined to be the best fit to the data. $A I C=$ Akaike information criterion; $\mathrm{BIC}=$ Bayesian information criterion; VLMR = Vuong-Lo-Mendell-Rubin likelihood ratio test; adjusted LMR = adjusted Lo-Mendell-Rubin likelihood ratio test.

Figure 1 illustrates the $z$-score standardized mean subscale scores (i.e., positive values indicate more use than the sample mean and negative values indicate less use than the sample mean) for each profile. Profiles were named according to their pattern of regulation. The profile with the largest group membership reported high levels of acceptance, cognitive reappraisal, and problem solving and low levels of avoidance, expressive suppression, and rumination. This pattern is hereafter characterized as the Adaptive regulation profile $(n=81 ; 46.02 \%)$ due to the putatively "adaptive" outcomes of these strategies in previous studies as well as this profile's relationship to psychological distress in the current study (see following analyses). The Accepting (with Suppression) profile ( $n=71 ; 40.34 \%)$ included participants who often regulated via acceptance and expressive suppression. The Nonaccepting regulators $(n=18 ; 10.23 \%)$ were characteristically distinct because of their extremely low levels of acceptance, coupled with moderately high use of avoidance and rumination. Individuals in the final profile, the Maladaptive regulation profile $(n=6 ; 3.41 \%)$, relied primarily on avoidance, expressive suppression, and rumination, and infrequently on acceptance, cognitive reappraisal, and problem solving. Again, this profile was named according to previously and currently demonstrated outcomes of these strategies (see following analyses).

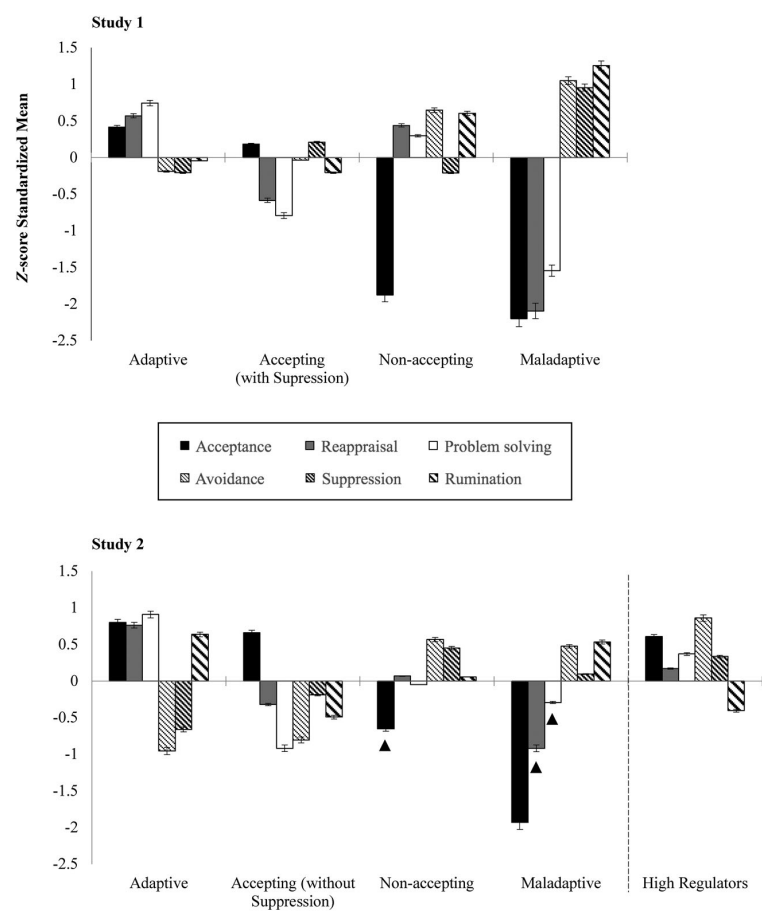

Figure 1. Profiles of emotion regulation. Between-study mean differences in strategy use across matched profiles (> 1 SD) are indicated by triangles beneath the corresponding strategy.

Psychological distress and emotion regulation profiles

To establish whether these profiles were meaningfully related to symptoms of psychological distress, a MANOVA was conducted to evaluate differences between the emotion regulation profiles on the outcomes of depression and anxiety. Multivariate results indicated significant differences in symptom reports, Wilks' $\lambda=0.66, F(6,342)=13.11, p<.001$, partial $\eta^{2}=.19$. Univariate and pairwise 
comparisons between profiles are reported below. Table 3 presents the means and standard deviations of symptoms, as well as the significant differences, associated with each profile.

Table 3. Psychopathological symptom differences across regulatory profiles.

\begin{tabular}{|l|l|l|l|l|}
\hline & M (SD) & & & \\
\hline & Depression & & $\begin{array}{l}\text { Generalized } \\
\text { anxiety }\end{array}$ & \\
\hline & Study 1 & Study 2 & Study 1 & Study 2 \\
\hline Adaptive ( $\left.\mathrm{n}_{1}=81 ; \mathrm{n}_{2}=29\right)$ & $8.72^{\mathrm{a}}(6.87)$ & $6.34^{\mathrm{a}}(5.54)$ & $8.07^{\mathrm{a}}(7.39)$ & $5.53^{\mathrm{a}}(6.31)$ \\
\hline $\begin{array}{l}\text { Accepting-with and without } \\
\left.\text { Suppression ( } \mathrm{n}_{1}=71 ; \mathrm{n}_{2}=32\right)\end{array}$ & $12.24^{\mathrm{b}}(7.62)$ & $6.23^{\mathrm{a}}(7.47)$ & $12.65^{\mathrm{b}}(11.91)$ & $7.68^{\mathrm{ab}}(9.30)$ \\
\hline High Regulators $\left(\mathrm{n}_{2}=26\right)$ & - & $9.04^{\mathrm{ab}}(7.81)$ & - & $8.08^{\mathrm{ab}}(8.75)$ \\
\hline Non-accepting $\left(\mathrm{n}_{1}=18 ; \mathrm{n}_{2}=42\right)$ & $16.50^{\mathrm{b}}(10.35)$ & $14.22^{\mathrm{bc}}(9.34)$ & $19.79^{\mathrm{bc}}(15.63)$ & $13.59^{\mathrm{bc}}(10.43)$ \\
\hline Maladaptive $\left(\mathrm{n}_{1}=6 ; \mathrm{n}_{2}=16\right)$ & $36.50^{\mathrm{c}}(14.02)$ & $18.75^{\mathrm{c}}(11.39)$ & $32.10^{\mathrm{c}}(10.88)$ & $21.31^{\text {cd }}(11.88)$ \\
\hline
\end{tabular}

Note: Within each column, means with differing superscripts are significantly different from each other $(p<.05)$. For each depression and anxiety, across-sample significant differences in symptom reports for matched profiles are bolded $(p<.05)$.

\section{Depression.}

Univariate results investigating depression symptom severity indicated significant differences across the profiles, $F(3,176)=26.05, p<.001$, partial $\eta^{2}=0.31$. Pairwise comparisons demonstrated that individuals with the lowest reported symptoms of depression had the Adaptive regulation profile; this group reported significantly fewer symptoms of depression than individuals in each of the other profiles [Accepting (with Suppression) $p=0.04$; Non-accepting $p=0.001$; Maladaptive $p<.001$ ]. Additionally, individuals in the Maladaptive regulation subgroup reported the most severe depressive symptoms, significantly greater than symptom reports in all other groups (all ps $<0.001$ ). There was no difference in depression scores between the Accepting-Suppressor and the Non-accepting profiles.

\section{Anxiety.}

Univariate results investigating reported anxiety level indicated significant group differences in symptoms across the emotion regulation profiles, $F(3,176)=14.44, p<.001$, partial $\eta^{2}=0.20$. Specifically, anxiety symptoms reported by the Adaptive profile were significantly less than anxiety symptoms associated with each of the other profiles [Accepting (with Suppression) $p=.048$; Nonaccepting, Maladaptive: $p<.001]$. Anxiety symptoms reported by individuals with the Maladaptive profile were significantly more severe than those reported in the Adaptive and Accepting (with Suppression) profile groups (both $p<.001$ ). Anxiety in the Maladaptive profile was not significantly different than the Non-accepting profile $(p=.09)$.

\section{Study 1 discussion}

These data demonstrate the existence of four meaningful, distinct emotion regulation profiles in an undergraduate sample: Adaptive, Accepting (with Suppression), Non-accepting, and Maladaptive. The overall pattern of findings from Study 1 demonstrates consistencies with previous undergraduate (Dixon-Gordon et al., 2015; Eftekhari et al., 2009) and community (Chesney \& Gordon, 2017) samples. Further, these findings provide additional support for the supposition that a random sample of 
participants will reliably contain two discrete subgroups of individuals who can be identified based on their regulatory profile. The first is an "adaptive" subgroup of individuals who describe themselves as having low levels of anxiety and depression, and whose regulatory profile is dominated by strategies that have been independently and in concert with other strategies associated with desirable psychosocial outcomes. The second is a "maladaptive" subgroup who report relatively high levels of anxiety and depression symptoms, and whose regulatory profile is primarily associated with strategies that have repeatedly shown undesirable psychosocial outcomes, again at the level of a single strategy and also in a framework with other strategies. Although the Maladaptive profile seen here is characterized by a small minority of the sample, this pattern of regulation is conceptually consistent with previous findings and is empirically supported by data-driven fit statistics. The two remaining profiles, Accepting (with Suppression) and Non-accepting, approximate patterns of regulation found in previous studies (Chesney \& Gordon, 2017; Dixon-Gordon et al., 2015). Still, there are specific aspects of these profiles, such as the marked difference between acceptance and all other strategies in the Non-accepting profile, that suggest these may be distinct profiles.

\section{Study 2}

Study 1 demonstrated the consistency of at least two profiles within the current literature, which had consistent, meaningful differences for psychological wellbeing. However, Study 1 also uncovered profiles that only partially replicate previous studies. Generating empirical support for the stability of additional profiles will allow us to better predict the relationships between regulatory strategies and psychological outcomes of specific strategy combinations (i.e., profiles). Study 2 was designed as a replication study, implementing the same measures and analyses in an independent sample. The goal was to minimize critical differences in methodology and demographic variables to effectively explore the consistency of the profiles from Study 1.

\section{Method}

\section{Participants.}

A sample of 147 undergraduate participants was recruited from the same urban, Midwestern university as Study 1. Recruitment methods, inclusion criteria, and assessment procedures were identical. Mean age for participants in this sample was 19.01 years $(S D=1.02)$, and $69 \%$ were female. Consistent with the previous sample, participants primarily identified as Caucasian $(n=114 ; 77.55 \%)$, and the remainder of the sample identified as Asian $(n=10 ; 6.80 \%)$, African American $(n=8 ; 5.44 \%)$, Hispanic/Latino ( $n=7 ; 4.76 \%$ ), or other (including multiracial individuals; $n=8,5.44 \%$ ). The sample from Study 2 did not differ significantly from the initial sample in Study 1 on demographics (i.e., age, gender, race), measures of psychological health (i.e., depression, anxiety), or any measured emotion regulation strategy (see Table 1 for means).

Materials.

The self-report assessments and method of administration used in Study 2 were identical to those used in Study 1. Means, standard deviations, and internal consistencies for the second sample are reported in Table 1. 


\section{Results}

Variable screening did not reveal any concerns regarding the normality of distributions, including outliers. However, idiosyncratic responding (i.e., multiple measures having all left-most sided Likert responses) was found for one participant. Therefore, this individual was removed prior to all analyses. Missing data procedures were identical to those in Study 1.

Descriptives and zero-order analyses

Analyses were conducted to determine whether measured demographic variables (i.e., age, gender, and race) were associated with either emotion regulation reports or the established regulatory profiles. Results indicated all non-significant findings (all $p s>.05$ ).

Profile solution

Paralleling the LPA from Study 1 (see Statistical Analyses section in Study 1 for details), a separate LPA was conducted for Study 2. Table 2 provides the fit statistics for all tested models. The six-profile solution was determined to provide the best fit to the data. Although the AIC, adjusted BIC, and entropy values argued for a seven-profile solution, the VLMR and adjusted LMR were non-significant, indicating that an additional profile was not warranted. In fact, the VLMR and adjusted LMR were significant for the six-profile solution, providing evidence for this model over others. The high entropy value (Clark \& Muthén, 2009) indicated that $79.8 \%$ of individuals are classified into the correct latent profile according to the six-profile model. The BIC (which tends to favor a solution with fewer groups; Dziak, Coffman, Lanza, \& Li, 2012) was lowest for the two-profile solution; however, given the superiority of multiple other statistics for the six-profile model, this solution was retained because it provided the best combination of fit statistics and theoretical support.

The $z$-score standardized mean subscale scores for each profile are shown in Figure 1. The Adaptive regulation profile $(n=29 ; 19.73 \%)$ consisted of participants who reported high levels of adaptive strategies and low levels of maladaptive strategies. The next profile included participants who often regulated via acceptance, and infrequently with any other measured strategy; this group was labeled as the Accepting (without Suppression) profile $(n=32 ; 21.77 \%)$. The High Regulators $(n=26$; $17.69 \%)$ profile was labeled as such due to this subgroup's frequent use of most strategies. Individuals in the Non-accepting regulation $(n=42 ; 28.57 \%)$ profile paralleled the profile from the first sample in their low levels of acceptance and high reliance on avoidance. The Maladaptive regulation profile ( $n=16 ; 10.88 \%$ ) relied primarily on the measured strategies that are typically considered maladaptive and infrequently on those considered adaptive.

The smallest proportion of the sample $(n=2 ; 1.36 \%)$ were Low Regulators; although this group only included two individuals, the inclusion of this profile provided a better fit to the data than other models (see Table 2). However, since few reliable conclusions may be drawn from a profile type with only two individuals, the Low Regulator subgroup was not included in any further analyses.

Psychological distress and emotion regulation profiles

Differences in symptom reports of depression and anxiety across the profiles were investigated by MANOVA, which yielded multivariate significance, Wilks' $\lambda=.72, F(8,278)=6.18, p<.001$, partial $\eta^{2}=0.15$. Univariate and pairwise comparisons for this sample follows below. Table 3 presents 
the means and standard deviations of depression and anxiety, as well as the significant differences for each profile in Study 2.

\section{Depression.}

Univariate results on depression symptoms indicated significant profile differences in symptom severity, $F(4,140)=10.20, p<.001, R^{2}=0.23$. Specifically, pairwise comparisons indicated that the severity of depression reported by the Adaptive and Accepting (without Suppression) profiles were statistically equivalent, and both were significantly less than the Non-accepting (both $p=.001$ ) and Maladaptive (both $p<.001$ ) profiles. Individuals with the Maladaptive profile also reported significantly higher depression than those with a High Regulator profile $(p=.003)$, but not those with a Nonaccepting profile $(p=.65)$.

\section{Anxiety.}

Univariate results indicated significant group differences in severity of reported anxiety symptoms across the emotion regulation profiles, $F(4,140)=9.66, p<.001, R^{2}=.22$. The Adaptive regulation profile was associated with the lowest level of anxiety, significantly lower than the Non-accepting $(p=.005)$ and Maladaptive profiles $(p<.001)$. The highest level of anxiety, reported by individuals with the Maladaptive profile, was significantly greater than those reported by all other groups (all $p<.001$ ), except the Non-accepting group $(p=.06)$.

\section{Cross-sample comparison}

To evaluate the consistency of emotion regulation patterns across studies, each profile from the first sample was coupled with a profile from the second sample to create matched profile pairs. Profiles were coupled according to similarity of regulation patterns. Statistical consistency between the profiles of Study 1 and Study 2 was evaluated by comparing the standardized means for each strategy across the matched profile pairs, and determining whether the differences were greater than 1 SD. The High Regulators profile was not present in the sample from Study 1, and therefore it was not matched in any profile pair. This resulted in four matched profile pairs: (1) the Adaptive profiles, (2) the Accepting profiles, (3) the Non-accepting profiles, and (4) the Maladaptive profiles. Individual strategies were compared across the two samples, within each pair. Only three comparisons evidenced differences greater than 1 SD (indicated with triangles in Figure 1). In the Non-accepting profiles, the degree of acceptance was lower in Study $1(z=-1.88)$ compared to Study $2(z=-0.65)$. In the Maladaptive profiles, both cognitive reappraisal and problem solving were used less in Study 1 (cognitive reappraisal: $z=-2.10$; problem solving: $z=-1.55$ ) compared to Study 2 (cognitive reappraisal: $z=-0.92$; problem solving: $z=-0.29$ ). Despite these three differences in magnitude across studies, it should be noted that the use of strategies relative to the mean was the same (i.e., both studies' Maladaptive regulators used cognitive reappraisal and problem solving less than the average, and both studies' Non-accepting regulators used acceptance less than the average). Thus, the current findings provide evidence for the similarity in the overall regulatory pattern for individuals with a particular profile.

Matched profile differences in psychological distress

Given the evidence that emotion regulation use is associated with differences in psychopathology, the current study also evaluated possible differences in symptom reports across matched profiles (differences bolded in Table 3). Four, one-way MANOVAs - one examining each of the matched profile pairs - were conducted to determine between-sample differences in the outcomes of depression and 
anxiety. For the matched Adaptive regulation profiles, multivariate results indicated no significant differences in symptom levels between the two samples, suggesting consistency between the matched profiles, $F(2,107)=1.79, p=.172$, partial $\eta^{2}=.03$. In the matched Accepting profiles, there was a significant difference between samples $F(2,100)=6.92, p=.002$, partial $\eta^{2}=.12$. Univariate tests indicated that the sample from Study 1 who were categorized as having an Accepting (with Suppression) profile reported higher levels of depression, $F(1,101)=13.86, p<.001$, partial $\eta^{2}=.12$, and anxiety, $F(1,101)=4.36, p=.039$, partial $\eta^{2}=.04$, than their counterparts in the Accepting (without Suppression) profile from Study 2. Analysis of the matched Non-accepting profiles samples yielded no significant differences in symptom reports, $F(2,57)=1.66, p=.200$, partial $\eta^{2}=.06$. The multivariate differences between the matched Maladaptive profiles, $F(2,19)=5.45, p=.013$, partial $\eta^{2}=.36$, were indicative of a significant difference in depression but not anxiety. Specifically, individuals from Study 2 with a Maladaptive profile reported more severe depressive symptoms than individuals with this profile in Study $1, F(1,20)=9.39, p=.006$, partial $\eta^{2}=.32$.

\section{Study 2 discussion}

The sample in Study 2 was collected to examine the replicability of the observed profiles from Study 1. LPA indicated that the sample from Study 2 was effectively categorized into six regulatory profiles: Adaptive, Accepting (without Suppression), High Regulators, Non-accepting, Maladaptive, and Low Regulators. Four of the profiles demonstrated marked consistency with those profiles found in Study 1 (Adaptive, Accepting, Non-accepting, and Maladaptive), with few differences in their use of the measured regulatory strategies. The conceptualization of these profiles was further supported by their associations with psychological health. For example, the greatest levels of depression and anxiety symptoms were observed in the maladaptive profiles, whereas the lowest

levels of symptoms were associated with the adaptive profiles. While there was variability in symptom severity between some of the profiles matched across Studies 1 and 2, the differences underscore the interplay between strategies (such as expressive suppression) and their influence on psychological health.

\section{General discussion}

While existing research has advanced our understanding about the contribution of singular emotion regulation strategies to psychological health, the current paper addresses uncertainties about the potential for consistency in how individuals combine multiple strategies to regulate emotion. These studies extend confidence in the argument for replicable emotion regulation profiles with a novel investigation that included six, frequently studied regulation strategies and identified regulatory profiles with an advanced person-centered analytical method. In Study 1, LPA established four distinct regulatory profiles in an undergraduate sample: Adaptive, Accepting (without Suppression), Nonaccepting, and Maladaptive. While each regulatory profile was based solely on typical emotion regulation use, the profiles exhibited meaningful relationships with symptoms of depression and anxiety. For example, the Adaptive profile was associated with fewer depressive symptoms and anxiety, while the Maladaptive profile was associated with greater symptoms. While the LPA conducted in Study 2's replication sample yielded a six-profile solution, four of the profiles matched those found in Study 1 and provided evidence for distinct, stable profiles characterized by: (1) high use of putatively adaptive strategies ("Adaptive"); (2) high use of putatively maladaptive strategies 
("Maladaptive"); ( 3) high use of acceptance ("Accepting"); and ( 4) low use of acceptance ("Nonaccepting"). These findings generally supported study hypotheses, which stated that profiles would be found, and replicated, that would tend towards adaptive strategies and psychological health, as well as toward maladaptive strategies and psychological distress. Hypotheses also proposed profiles that would not be distinguishable by their preference for either adaptive or maladaptive strategies and could be associated with variable levels of psychological wellness. These profiles are exemplified by the Accepting and Non-accepting profiles. In a small number of cases, there were magnitude differences in how often a particular strategy was used across matched profiles, such as cognitive reappraisal and problem solving in the Maladaptive profiles. Still, the overall pattern of use for the system of strategies presented here demonstrates considerable stability in these four profiles, and supports findings from previous studies as well (Chesney \& Gordon, 2017; Dixon-Gordon et al., 2015; Eftekhari et al., 2009).

When examining differences in the patterns of strategies, and their relationships to psychological health, variability in acceptance appeared to be a key factor. Indeed, the greatest variability between the two studies resulted from the Accepting profiles. The profile that had high levels of acceptance, as well as elevated use of suppression, had more symptoms of depression and anxiety than the profile that relied primarily on acceptance alone. This highlights the interplay between strategies as an important component in psychological wellbeing (e.g., Aldao \& Nolen-Hoeksema, 2012). Additionally, across profiles levels of psychopathological symptoms increase as reported use of acceptance reliably decreases. This is most evident when considering the Adaptive and Accepting profiles. Acceptance use is above average in both profiles and these two profiles exhibited low level of reported psychopathological symptoms relative to other profiles. This parallels literature suggesting that acceptance is vital in predicting greater functioning and positive outcomes for individuals suffering from depression (Garnefski \& Kraaij, 2006b; McCracken \& Eccleston, 2005).

Similarly, suppression appears to play a critical role in the association of emotion regulation strategies and symptoms of psychological distress. The Accepting without Suppression profile was statistically equivalent to the Adaptive profile in terms of depression and anxiety. However, when the Accepting profile included above average levels of suppression, then it was related to significantly more psychological distress than the adaptive profile. This suggests that while acceptance may be a key factor when examining adaptive outcomes, suppression may dampen those effects. This is consistent with previous literature demonstrating maladaptive strategies' stronger effect size on psychopathological symptoms as compared to adaptive strategies (Aldao et al., 2010). Furthermore, this nuance of high and low suppression coupled with acceptance emphasizes the utility of evaluating strategy use with profiles as opposed to examining any one strategy individually.

When examining the emotion regulation profiles present in the sample from Study 2, two profiles were present in addition to those found in Study 1 . These profiles characterized individuals who globally regulated less than the average person, Low Regulators, or more than average person, High Regulators. Although the Low Regulators profile was comprised of only two individuals, this profile has been observed in other studies (Dixon-Gordon et al., 2015; Eftekhari et al., 2009), which suggests that this regulatory style is worthy of future study. The High Regulator profile parallels the Multi-strategy profile found in the replication study by De France and Hollenstein (2017), as well as the High Regulators group presented by Dixon-Gordon et al. (2015). This regulatory pattern also warrants future 
study, as there continues to be disagreement with regards to the adaptability of a regulatory pattern characterized by frequent reliance on nearly all measured strategies. The current findings are aligned with work (Dixon-Gordon et al., 2015) showing greater levels of anxiety and depression for high regulators when compared with individual's whose profiles are characterized by predominantly adaptive strategies (though these differences were non-significant herein). This is contrasted with findings showing relatively low symptom reports for multi-strategy users (De France \& Hollenstein, 2017).

One deviation of the current results from previous studies was the uncharacteristically elevated report of rumination for the Adaptive profile - a profile otherwise dominated by adaptive strategies - in Study 2. Rumination has been identified as a potentially multidimensional construct comprised of adaptive and maladaptive components. Thus, it is possible that the components of rumination characterized in the Adaptive profile here are more of reflective pondering (Joormann, Dkane, \& Gotlib, 2006), or present-moment experiential thought processes (Watkins \& Teasdale, 2004), associated with positive psychological outcomes. Additionally, this finding supports the conclusion that person-centered approaches can account for complex relationships between strategies and clarify when a strategy is helpful or unhelpful. For instance, Aldao and Nolen-Hoeksema (2012) propose and provide empirical support (Aldao \& Nolen-Hoeksema, 2012) for the compensatory hypothesis, which suggests that the use of adaptive strategies is especially beneficial when maladaptive strategies are also being used because the negative effects of maladaptive strategies are dampened by more adaptive approaches. In other words, individuals using maladaptive strategies would be predicted to report low psychological distress, as long as they were also using adaptive strategies. This was the case for Study 2 individuals with the Adaptive profile, for whom significant reliance on acceptance, cognitive reappraisal, and problem solving may be compensating for the use of rumination, therefore overriding the negative effects of this strategy and resulting in minimal psychological distress. Interestingly, this compensatory relationship does not appear to hold for the Accepting with Suppression profile, in which the above average use of acceptance and suppression is related to increased symptom levels. The presence of these complex dynamics between strategies necessitates additional studies that continue to investigate their bidirectional influences upon one another, as well as their downstream effects on psychosocial outcomes.

\section{Limitations.}

The current studies have some limitations. First, the reliance on self-report measures to assess use of emotion regulation strategies is not immune to bias, including social desirability and inflated shared variance between the measures. Additionally, the current findings represent a cross-sectional assessment of general regulation habits. Therefore, these data do not allow for conclusions regarding causality between use of a particular profile and psychological health, nor do they allow for the evaluation of whether profiles are consistent across specific contexts. Future studies may utilize context-dependent and/or longitudinal study designs to begin appropriately addressing these important limitations. Although the two studies presented here include independent samples, both groups were collected from the same population of undergraduates. This form of replication offers a critical first step in validating the existence of emotion regulation profiles in college-aged individuals. Future studies may further the current research by implementing these methods in samples collected from diverse sites. 
Finally, two of the profiles had particularly small numbers - Low Regulators (Study 2) and Maladaptive regulators (Study 1). We chose not to conduct additional analyses on the Low regulator profile $(n=2)$, but we did include the Maladaptive profile $(n=6)$. Empirically, maladaptive profiles have consistently been observed in other studies (e.g., Dixon-Gordon et al., 2015) and arguably merit inclusion here. Further, given that this profile is generally comprised of individuals with impairing psychological symptoms, and that the current data are on a non-clinical sample of undergraduates, it is expected that fewer individuals would endorse a profile of "maladaptive" strategy use. Still, some of the current group sizes introduce potential violations of homogeneity of variance and threaten statistical power in some difference tests. Most of the difference tests in the current studies had adequate power (i.e., greater than 0.9); however, the tests examining the differences in psychological distress for matched Adaptive and Non-accepting profiles did evidence low observed power and were non-significant. Therefore, these null findings should be interpreted with caution. A larger sample size may reconcile the small number of participants in these profiles, as well as address the concern that the current sample may not have allowed for the identification of additional important, but perhaps not highly prevalent, profiles.

\section{Clinical Implications and Future Directions.}

In addition to the meaningful contributions that the current studies add to the research on regulatory profiles, and the links between particular patterns to depression and anxiety, they also offer a number of implications that translate to mental health treatment. Understanding the nuanced differences in profiles, and how they relate to psychopathology, can assist clinicians in providing meaningful strategies to patients. For example, findings presented by the current two studies support psychotherapeutic techniques aimed at increasing acceptance and reducing suppression of emotion (e.g., Acceptance and Commitment Therapy; Hayes \& Wilson, 1994).

The current studies present a number of questions for future research, including the need for longitudinal studies that can assess the consistency of emotion regulation profiles over an individual's lifetime. Further, a considerable number of demographic (e.g., age and gender; Nolen-Hoeksema \& Aldao, 2011), as well as situational factors (e.g., past traumatic events; Bardeen, Kumpula, \& Orcutt, 2013), have been shown to influence regulation and affect the relationship between emotion regulation and psychological health. Therefore, it will be important for future studies to continue assessing profile consistencies, and discrepancies, in distinct contexts and with replicable samples. This will allow for the field to establish variables that may contribute to risk or protective factors in managing emotional experiences.

\section{Disclosure statement}

No potential conflict of interest was reported by the authors.

\section{References}

Akaike, H. ( 1973 ). Information theory and an extension of the maximum likelihood principle. In B. N. Petrov \& F. Csáki (Eds.), Proceedings of the second international symposium on information theory (pp. 267 - 281 ). Budapest : Akadémiai Kiadó. 
Aldao, A. ( 2013 ). The future of emotion regulation research: Capturing context. Perspectives on Psychological Science, 8 ( 2 ), 155 - 172. doi: 10.1177/1745691612459518

Aldao, A., Jazaieri, H., Goldin, P. R., \& Gross, J. J. ( 2014 ). Adaptive and maladaptive emotion regulation strategies: Interactive effects during CBT for social anxiety disorder. Journal of Anxiety Disorders, 28 ( 4 ), 382 - 389. doi: 10.1016/j.janxdis.2014.03.005

Aldao, A., \& Nolen-Hoeksema, S. ( 2012 ). When are adaptive strategies most predictive of psychopathology? Journal of Abnormal Psychology, 121 ( 1 ), 276 - 281. doi: 10.1037/a0023598

Aldao, A., Nolen-Hoeksema, S., \& Schweizer, S. ( 2010 ). Emotion-regulation strategies across psychopathology: A meta-analytic review. Clinical Psychology Review, 30 ( 2 ), 217 - 237. doi: 10.1016/j.cpr.2009.11.004

Aldao, A., \& Tull, M. T. ( 2015 ). Putting emotion regulation in context. Current Opinion in Psychology, 3, 100 - 107. doi: 10.1016/j.copsyc.2015.03.022

Bardeen, J. R., Kumpula, M. J., \& Orcutt, H. K. ( 2013 ). Emotion regulation difficulties as a prospective predictor of posttraumatic stress symptoms following a mass shooting. Journal of Anxiety Disorders, 27 ( 2 ), 188 - 196. doi: 10.1016/j.janxdis.2013.01.003

Beck, A. T., Epstein, N., Brown, G., \& Steer, R. A. ( 1988 ). An inventory for measuring clinical anxiety: Psychometric properties. Journal of Consulting and Clinical Psychology, 56 ( 6 ), 893 - 897 . doi: 10.1037/0022-006X.56.6.893

Beck, A. T., Steer, R. A., \& Brown, G. K. ( 1996 ). Beck Depression Inventory-II (BDI-II). San Antonio, TX : Psychological Corporation.

Berlin, K. S., Williams, N. A., \& Parra, G. R. ( 2014 ). An introduction to latent variable mixture modeling (Part 1): Overview and cross-sectional latent class and latent profile analyses. Journal of Pediatric Psychology, 39 ( 2 ), 174 - 187. doi: 10.1093/jpepsy/jst084

Brans, K., Koval, P., Verduyn, P., Lim, Y. L., \& Kuppens, P. ( 2013 ). The regulation of negative and positive affect in daily life. Emotion, 13 ( 5 ), 926 - 939. doi: 10.1037/a0032400

Celeux, G., \& Soromenho, G. ( 1996 ). An entropy criterion for assessing the number of clusters in a mixture model. Journal of Classification, 13 ( 2 ), 195 - 212. doi: 10.1007/BF01246098

Chesney, S. A., \& Gordon, N. S. ( 2017 ). Profiles of emotion regulation: Understanding regulatory patterns and the implications for posttraumatic stress. Cognition and Emotion, 31 ( 3 ), 598 606. doi: 10.1080/02699931.2015.1126555

Clark, S. L., \& Muthén, B. ( 2009 ). Relating latent class analysis results to variables not included in the analysis. Retrieved from https://www.statmodel.com/download/relatinglca.pdf

De France, K., \& Hollenstein, T. ( 2017 ). Assessing emotion regulation repertoires: The regulation of emotion systems survey. Personality and Individual Differences, 119, 204 - 215. doi: 10.1016/j.paid.2017.07.018

Dixon-Gordon, K. L., Aldao, A., \& De Los Reyes, A. ( 2015 ). Repertoires of emotion regulation: A person-centered approach to assessing emotion regulation strategies and links to psychopathology. Cognition and Emotion, 29 ( 7 ), 1314 - 1325. doi: 10.1080/02699931.2014.983046

Doré, B. P., Silvers, J. A., \& Ochsner, K. N. ( 2016 ). Toward a personalized science of emotion regulation. Social and Personality Psychology Compass, 10 ( 4 ), 171 - 187. doi: $10.1111 /$ spc3.12240 
Dziak, J. J., Coffman, D. L., Lanza, S. T., \& Li, R. ( 2012 ). Sensitivity and specificity of information criteria. The Methodology Center and Department of Statistics, Penn State, The Pennsylvania State University, 16 ( 30 ), 1 - 30.

Eftekhari, A., Zoellner, L. A., \& Vigil, S. A. ( 2009 ). Patterns of emotion regulation and psychopathology. Anxiety, Stress, \& Coping, 22 ( 5 ), 571 - 586. doi: 10.1080/10615800802179860

Gabriel, A. S., Daniels, M. A., Diefendorff, J. M., \& Greguras, G. J. ( 2015 ). Emotional labor actors: A latent profile analysis of emotional labor strategies. Journal of Applied Psychology, 100 ( 3 ), 863 - 879. doi: 10.1037/a0037408

Garnefski, N., \& Kraaij, V. ( 2006a ). Cognitive emotion regulation questionnaire-development of a short 18-item version (CERQ-short). Personality and Individual Differences, 41 ( 6 ), 1045 - 1053. doi: 10.1016/j.paid.2006.04.010

Garnefski, N., \& Kraaij, V. ( 2006b ). Relationships between cognitive emotion regulation strategies and depressive symptoms: A comparative study of five specific samples. Personality and Individual Differences, 40 ( 8 ), 1659 - 1669. doi: 10.1016/j.paid.2005.12.009

Gratz, K. L., \& Roemer, L. ( 2004 ). Multidimensional assessment of emotion regulation and dysregulation: Development, factor structure, and initial validation of the difficulties in emotion regulation scale. Journal of Psychopathology and Behavioral Assessment, 26 ( 1 ), 41 - 54. doi: 10.1023/B:JOBA.0000007455.08539.94

Gross, J. J. ( 2002 ). Emotion regulation: Affective, cognitive, and social consequences. Psychophysiology, 39 ( 3 ), 281 - 291. doi: 10.1017.S0048577201393198

Gross, J. J., \& John, O. P. ( 2003 ). Individual differences in two emotion regulation processes: Implications for affect, relationships, and well-being. Journal of Personality and Social Psychology, 85 ( 2 ), 348 - 362. doi: 10.1037/0022-3514.85.2.348

Hayes, S. C., \& Wilson, K. G. ( 1994 ). Acceptance and commitment therapy: Altering the verbal support for experiential avoidance. The Behavior Analyst, 17 ( 2 ), 289 - 303. doi: 10.1007/BF03392677

Heiy, J. E., \& Cheavens, J. S. ( 2014 ). Back to basics: A naturalistic assessment of the experience and regulation of emotion. Emotion, 14 ( 5 ), 878 - 891. doi: 10.1037/a0037231

Holahan, C. J., Moos, R. H., Holahan, C. K., Brennan, P. L., \& Schutte, K. K. ( 2005 ). Stress generation, avoidance coping, and depressive symptoms: A 10-year model. Journal of Consulting and Clinical Psychology, 73 ( 4 ), 658 - 666. doi: 10.1037/0022-006X.73.4.658

Joormann, J., Dkane, M., \& Gotlib, I. H. ( 2006 ). Adaptive and maladaptive components of rumination: Diagnostic specificity and relation to depressive biases. Behavior Therapy, 37 ( 3 ), 269 - 280. doi: 10.1016/j.beth.2006.01.002

Kashdan, T. B., Young, K. C., \& McKnight, P. E. ( 2012 ). When is rumination an adaptive mood repair strategy? Day-to-day rhythms of life in combat veterans with and without posttraumatic stress disorder. Journal of Anxiety Disorders, 26 ( 7 ), 762 - 768. doi: 10.1016/j.janxdis.2012.07.004

Kass, R. E., \& Raftery, A. E. ( 1995 ). Bayes factors. Journal of the American Statistical Association, 90 ( 430 ), 773 - 795. doi: 10.1080/01621459.1995.10476572

Keng, S.-L., Robins, C. J., Smoski, M. J., Dagenbach, J., \& Leary, M. R. ( 2013 ). Reappraisal and mindfulness: A comparison of subjective effects and cognitive costs. Behaviour Research and Therapy, 51 ( 12 ), 899 - 904. doi: 10.1016/j.brat.2013.10.006

Lazarus, R. S. ( 1991 ). Emotion and adaptation. New York, NY : Oxford University Press. 
Le, B. M., \& Impett, E. A. ( 2013 ). When holding back helps: Suppressing negative emotions during sacrifice feels authentic and is beneficial for highly interdependent people. Psychological Science. doi: 10.1177/0956797613475365

Lo, Y., Mendell, N. R., \& Rubin, D. B. ( 2001 ). Testing the number of components in a normal mixture. Biometrika, 88 ( 3 ), 767 - 778. doi: 10.1093/biomet/88.3.767

McCracken, L. M., \& Eccleston, C. ( 2005 ). A prospective study of acceptance of pain and patient functioning with chronic pain. Pain, 118 ( 1-2 ), 164 - 169. doi: 10.1016/j.pain.2005.08.015

Moos, R. H. ( 1993 ). Coping responses inventory: Adult professional manual. Lutz, FL : Psychological Assessment Resources, Inc.

Muthén, L. K., \& Muthén, B. O. ( 1998-2017 ). Mplus user's guide ( 8th ed. ). Los Angeles, CA : Muthén \& Muthén.

Nolen-Hoeksema, S., \& Aldao, A. ( 2011 ). Gender and age differences in emotion regulation strategies and their relationship to depressive symptoms. Personality and Individual Differences, 51 ( 6 ), 704 - 708. doi: 10.1016/j.paid.2011.06.012

Nylund, K. L., Asparouhov, T., \& Muthén, B. O. ( 2007 ). Deciding on the number of classes in latent class analysis and growth mixture modeling: A Monte Carlo simulation study. Structural Equation Modeling: A Multidisciplinary Journal, 14 ( 4 ), 535 - 569. doi: 10.1080/10705510701575396

Pastor, D. A., Barron, K. E., Miller, B., \& Davis, S. L. ( 2007 ). A latent profile analysis of college students' achievement goal orientation. Contemporary Educational Psychology, 32 ( 1 ), 8 - 47. doi: 10.1016/j.cedpsych.2006.10.003

Schwarz, G. ( 1978 ). Estimating the dimension of a model. The Annals of Statistics, 6 ( 2 ), 461 - 464. doi: 10.1214/aos/1176344136

Sclove, S. L. ( 1987 ). Application of model-selection criteria to some problems in multivariate analysis. Psychometrika, 52 ( 3 ), 333 - 343. doi: 10.1007/BF02294360

Van Eck, K., Warren, P., \& Flory, K. ( 2017 ). A variable-centered and person-centered evaluation of emotion regulation and distress tolerance: Links to emotional and behavioral concerns. Journal of Youth and Adolescence, 46 ( 1 ), 136 - 150. doi: 10.1007/s10964-016-0542-y

Watkins, E., \& Teasdale, J. D. ( 2004 ). Adaptive and maladaptive self-focus in depression. Journal of Affective Disorders, 82 ( 1 ), 1 - 8. doi: 10.1016/j.jad.2003.10.006 